\title{
Chemometric Analysis of UV-Visible Spectral Fingerprints for the Discrimination and Quantification of Clinical Anthracycline Drug Preparation Used in Oncology
}

\author{
Aimen El Orche $\mathbb{D D}^{1}{ }^{1}$ Casimir Adade Adade, ${ }^{2}$ Hafid Mefetah, ${ }^{3}$ Amine Cheikh, \\ Khalid Karrouchi, ${ }^{2}$ Miloud El Karbane, ${ }^{2}$ and Mustapha Bouatia $\left(^{2}\right.$ \\ ${ }^{1}$ Laboratory of Chemical Processes and Applied Materials, Faculty of Science and Technology, Sultan Moulay Slimane University, \\ Beni-Mellal, Morocco \\ ${ }^{2}$ Team of Formulation and Quality Control of Health Products, Faculty of Medicine and Pharmacy, \\ Mohammed V University in Rabat, Morocco \\ ${ }^{3}$ Rabat Pediatrics Hospital, Ibn Sina University Hospital Center, Rabat, Morocco \\ ${ }^{4}$ Departement of Pharmacy, Faculty of Pharmacy, Abulcasis University, Rabat, Morocco
}

Correspondence should be addressed to Mustapha Bouatia; m.bouatia@um5s.net.ma

Received 29 January 2021; Revised 12 April 2021; Accepted 29 April 2021; Published 7 May 2021

Academic Editor: Nahide Gulsah Deniz

Copyright (C 2021 Aimen El Orche et al. This is an open access article distributed under the Creative Commons Attribution License, which permits unrestricted use, distribution, and reproduction in any medium, provided the original work is properly cited.

\begin{abstract}
In clinical treatment, the analytical quality assessment of the delivery of chemotherapeutic preparations is required to guarantee the patient's safety regarding the dose and most importantly the appropriate anticancer drug. On its own, the development of rapid analytical methods allowing both qualitative and quantitative control of the formulation of prepared solutions could significantly enhance the hospital's workflow, reducing costs, and potentially providing optimal patient care. UV-visible spectroscopy is a nondestructive, fast, and economical technique for molecular characterization of samples. A discrimination and quantification study of three chemotherapeutic drugs doxorubicin, daunorubicin, and epirubicin was conducted, using clinically relevant concentration ranges prepared in $0.9 \% \mathrm{NaCl}$ solutions. The application of the partial least square discriminant analysis PLS-DA method on the UV-visible spectral data shows a perfect discrimination of the three drugs with a sensitivity and specificity of $100 \%$. The use of partial least square regression PLS shows high quantification performance of these molecules in solution represented by the low value of root mean square error of calibration (RMSEC) and root mean square error of cross validation (RMSCECV) on the one hand and the high value of $R$-square on the other hand. This study demonstrated the viability of UV-visible fingerprinting (routine approach) coupled with chemometric tools for the classification and quantification of chemotherapeutic drugs during clinical preparation.
\end{abstract}

\section{Introduction}

Currently, oncology treatment studies have become very important. Unfortunately, the number and cost of new anticancer drugs are still higher than ever before. This constitutes an economic challenge for countries with limited resources [1].

Daunorubicin, doxorubicin, and epirubicin belong to the family of anthracyclines used primarily as first-line drugs in combination with other chemotherapeutic drugs for a wide range of cancers, including breast cancer, bladder cancer, soft tissue and bone sarcomas, malignant lymphomas, and acute lymphocytic leukemia [2]. However, these therapeutic compounds have serious side effects: cardiotoxicity and myelosuppression are dependent on the dose [3]. These three anthracycline therapeutic molecules have similar molecular properties, which pose a great challenge for their discrimination.

The preparation of these anticancer drugs represents an important step in oncological treatment. Generally, this preparation has to comply with several rules and objectives; the safety of all personnel involved in terms of exposure, patient safety, and good production practices [4]. This 
preparation work must be carried out in a well-controlled atmosphere, with standardization of practices and the establishment of an effective system for monitoring and checking preparation quality $[5,6]$. However, there remains a residual proportion of major or minor errors leading to nonconformity, despite all the precautions taken in a centralized unit.

In order to guarantee the pharmaceutical and chemical quality of the preparation, several strategies of quality control have been elaborated to make sure that the right drug is delivered at the right concentration [7].High-performance liquid chromatography coupled with UV (HPLC/UV) has been developed for proper drug preparation in chemotherapy and is considered one of the earliest methods used for monitoring such drugs [8-11]. In addition, other spectroscopic methods, such as UV-visible, near infrared, and Raman [12-15], have also been applied for the quality control of drug preparation in chemotherapy.

High-performance liquid chromatography coupled with spectrometry is a well-established approach for monitoring hospital chemotherapy preparations [16]. Generally, these analytical methods based on chromatography require the use of reagents which are sometimes expensive and harmful to the environment, time consuming to carry out an analytical test, and require qualified operators. For this reason, the development of fast and environmentally friendly methods constitutes a great challenge in the field of analytical chemistry. Recently, new technology has been emerged to satisfy this demand, and these methods are based on the combination of nondestructive spectroscopic methods with the recognition algorithms such as PLS-DA, PLS, and support vector machine for the classification between groups of individuals and the quantification of their content in a given matrix according to spectral data [17-19].

Today, UV-visible spectroscopy combined with chemometric classification algorithms is widely used to develop rapid methods for drug quality control [20] and well established in the pharmaceutical industry to identify raw materials prior to production, falsified drugs, and also in process analytical technology.

This study focused on three chemotherapeutic drugs that are very similar in physicochemical properties and are difficult to discriminate: epirubicin, doxorubicin, and daunorubicin. These drugs are anthracyclines and antitumor antibiotics consisting of a planar anthraquinone ring attached to a carbohydrate containing an amino group. Doxorubicin is a natural product obtained from Streptomyces galilaeus while epirubicin is its semisynthetic analogue which differs only in its stereochemistry. These molecules function by intercalating DNA strands and leading to the formation of complexes that inhibit DNA and RNA synthesis. Daunorubicin is an anticancer drug used in the treatment of acute myeloblastic leukemia and acute lymphoblastic leukemia. Daunorubicin (DNR) derived from the fermentation of Streptomyces strains and was the first anthracycline used in clinical practice $[15,21]$. It is an agent that causes the blockage of DNA replication and the accelerated cell death of cancer cells. This drug is very often formulated in our hospital for the treatment of cancer diseases.

The aim of this study was to confirm the ability of UVvisible spectroscopy combined with multivariate data analy- sis such as PCA, PLS-DA, and PLS to monitor three anthracycline drugs at different concentrations, in order to examine the ability of this technology to discriminate and quantify these three drugs, which are considered among the most crucial to distinguish between them due to their similar chemical structures and their low concentrations.

\section{Materials and Methods}

Figure 1 shows an illustration of the molecular structure of daunorubicin, doxorubicin, and epirubicin that belongs to the anthracycline family used in chemotherapy. These three molecules are used to treat various types of cancer. These drugs have a similar molecular structure, as well as common physicochemical properties, which makes their discrimination difficult in the clinical chemotherapy preparation.

2.1. Sample Preparation. Several commonly used anthracyclines (Ddunorubicin, doxorubicin, and epirubicin) have been diluted at different concentrations with $0.9 \%$ sodium chloride which are usually used for this type of reconstitutions. Subsequently, they were analyzed directly using a double-beam spectrophotometer UV-visible Perkin-Elmer Lambda 12, at a wavelength ranging from 350 to $800 \mathrm{~nm}$.

The study was performed at different concentrations corresponding to (0.001-0.0064 $\mathrm{mg} / \mathrm{mL}$ for doxorubicin, 0.006 $0.077 \mathrm{mg} / \mathrm{mL}$ for daunorubicin and $0.006-0.083 \mathrm{mg} / \mathrm{mL}$ for epirubicin). A calibration model was developed by PLS-DA, and then crossvalidation was used to validate these models. For the construction of the PLS-DA and PLS regression model, a database of 52 samples has been used (22 of doxorubicin, 15 of daunorubicin, and 15 of epirubicin).

2.2. Multivariate Data Analysis. Chemometrics, also known as multivariate data analysis, is the science that implements optimal mathematical and statistical approaches to process chemical data. Chemometrics involves experiment design upstream and data processing to obtain valuable information once measurements have been taken. The growing need for chemometric tools comes especially through the development of analytical equipment that provides big amounts of complex data [22].

This scientific field comprises a wide variety of statistical methods, aimed at analyzing numerous data sets especially spectral data such as infrared spectroscopy, fluorescence, and UV-visible spectroscopy to achieve several objectives.

2.3. Partial Least Squares Regression. Partial least squares (PLS) regression is a chemometric technique often used for data modeling. PLS allows the reduction of predictors to a smaller set of noncorrelated components called latent variables (LVs) and performs the least squares regression on these main principal components (LV). The PLS feature is especially useful when the initial variables (predictors) are strongly correlated, or when the number of predictors exceeds the number of observations. PLS regression can be summarized in two related steps:

Dimensionality reduction of the $X$ (initial data matrix) and $Y$ (response matrix) matrix into latent variables using score projections. Then, the incorporation of a vector, known 

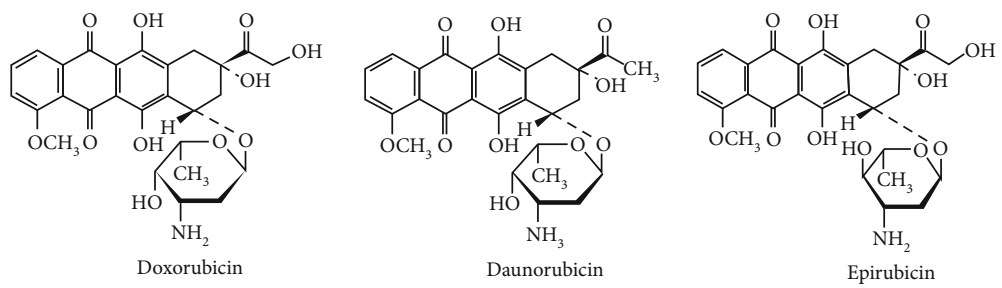

FIGURE 1: Molecular structure representation of the three drugs of the anthracycline family.

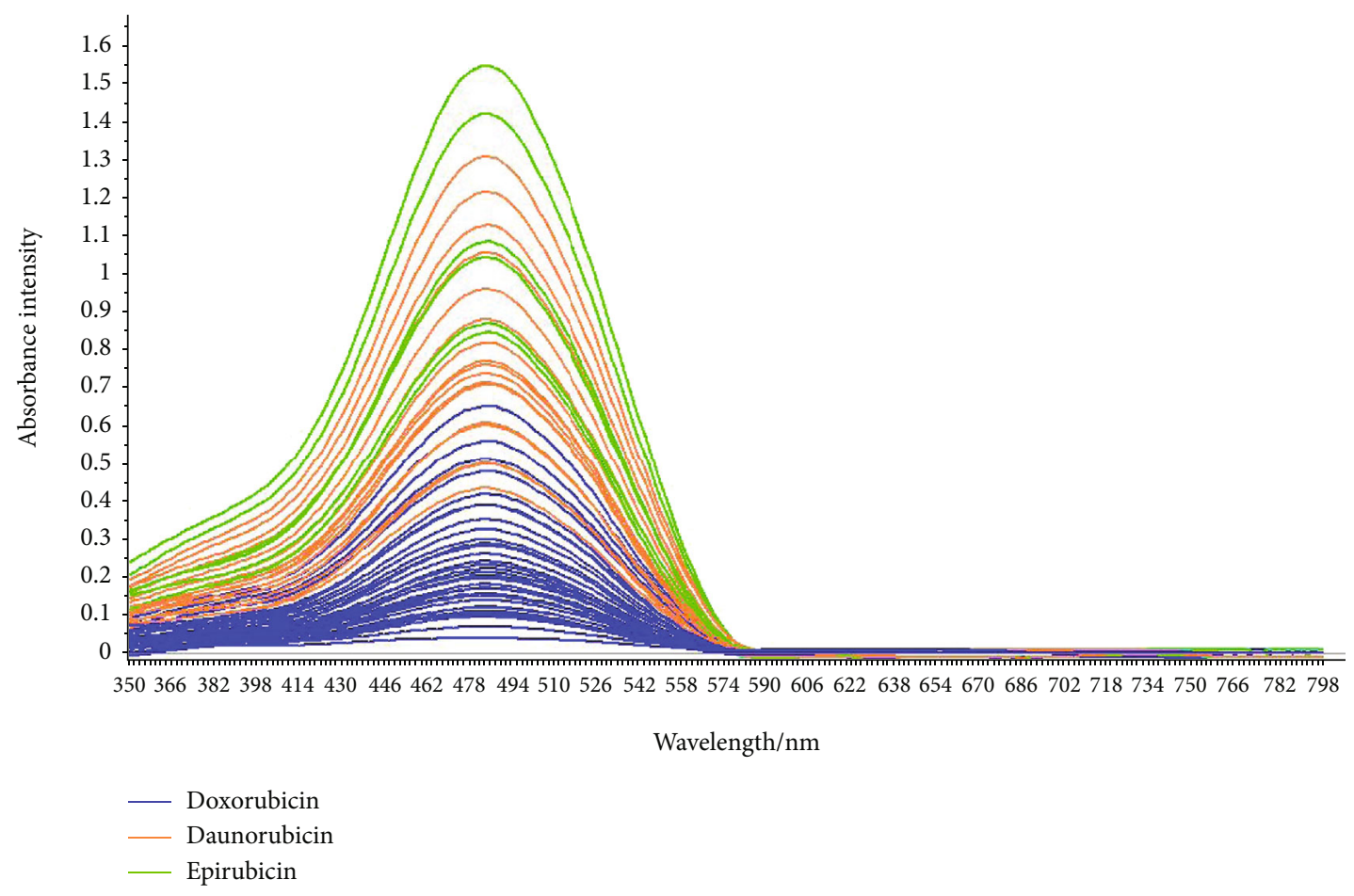

FIGURE 2: Graphical representation of the UV-visible absorption spectra of the three molecules.

as weight $(\mathrm{w})$, relates to the two latent variable matrices and providing the covariance between the $X$ and $Y$ scores as an optimization criterion.

2.4. Partial Least Squares Discriminant Analysis. The partial least squares (PLS) algorithm was initially implemented for the regression task and subsequently evolved into a well-known classification method known as PLS discriminant analysis (PLS-DA). PLS-DA integrates dimensionality reduction and discriminant analysis into a unique algorithm and is especially applicable to high-dimensional data modeling [23].

Traditionally, PLS has been proposed to deal with continuous variables (i.e., a stain) in regression. But in the cluster task, the output variables will be always categorical. Hence, the first step in PLS-DA modeling is to recode the categorical variables (i.e., ordinal or nominal) into continuous (i.e., numerical) variables $[23,24]$.

Two principal algorithms can be applied to perform PLS regression: an iterative procedure is known as the NIPALS algorithm (nonlinear iterative partial least squares) [25], or a direct method, known as the SIMPLS algorithm [26]. In this study, the PLS regression was performed using the NIPALS algorithm.

2.5. Software. All data analysis of UV-visible spectra and chemometric applications, PCA, PLS-DA, and PLS regression, were performed using Unscrambler software, version 10.4 camo analytic.

\section{Results and Discussion}

According to the UV-visible spectrum of the molecule, we notice that these three molecules absorb in a similar wavelength region with maximum absorption in $480 \mathrm{~nm}$ as shown in Figure 2, and this is explained by the high number of chromophores present in the three drugs (Figure 1). However, observable differences have been noticed in the spectral intensity, but this difference is due to the difference between the preparation concentrations of these molecules, which are prepared in different concentrations.

Due to the similarity shown in the molecular structure of the three molecules belonging to the anthracycline family, it is very difficult to discriminate between these three molecules 


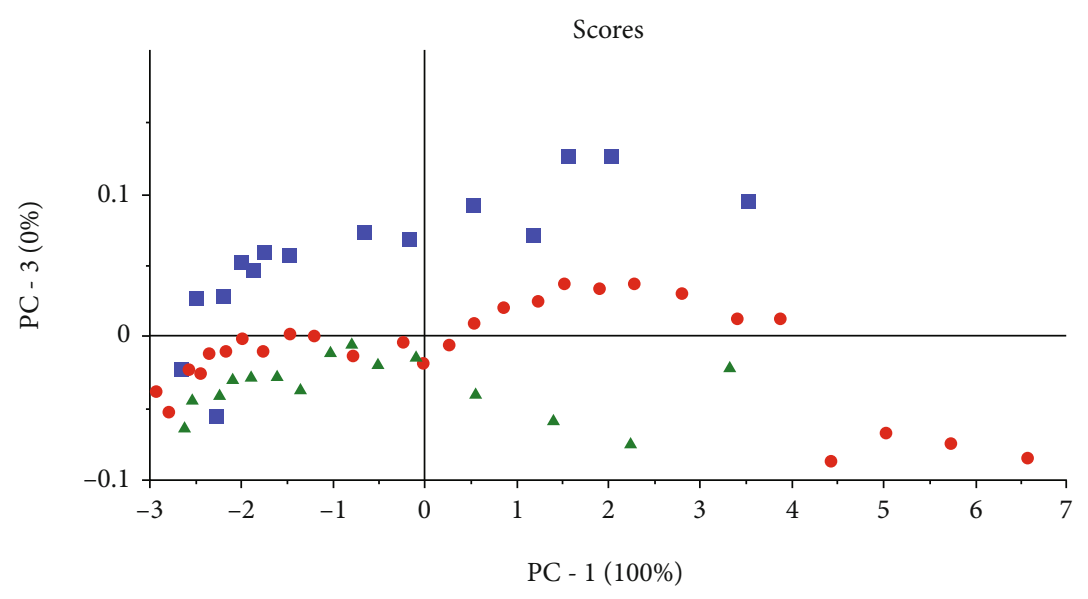

- DAUNORUBICIN

- DOXORUBICIN

- EPIRUBICIN

Figure 3: PCA score plot results of two principal components (PC1-PC3).

during the pharmaceutical preparation using UV-Visible spectroscopy.

3.1. Principal Component Analysis (PCA). In Figure 2, the resulting UV-Visible spectra show no significant differences between the three drugs, and only differences in absorption intensity were visually observable. The observation of spectral differences between the samples was difficult to see with the naked eye when these drugs are prepared in close or similar concentrations, and for this reason, chemometric statistical methods can be used to help observe the variability of the spectral data. Chemometric statistical methods include PCA. This method can be used to cluster samples and observe clustering relationships between samples based on differences in the UV-visible spectral data of each sample. The PCA method was mainly used to reduce the dimensionality of data in order to obtain a better visualization about individual's similarity, group's classification, and variable correlation.

PCA analysis revealed that the variability distribution was mainly given by the first principal component PC1, which represents $100 \%$ of total variability. The use of the score plot (Figure 3) demonstrates that there is a classification between the three drugs, and this classification was generally considered very weak, due to the similar physicochemical properties of the drugs.

PCA analysis shows that the first component gives useful information about the concentration of these drugs, and samples of high concentration contribute substantially and positively with the $\mathrm{PC} 1$ axis while samples of low concentration contribute negatively with the PC1 axis.

3.2. Discriminant Analysis PLS-DA. In order to successfully develop classification models capable of determining the class of the three drugs, chemometric classification tools have been applied to the spectral data. In this study, PLS-DA was used to discriminate the three chemotherapeutic drugs. The

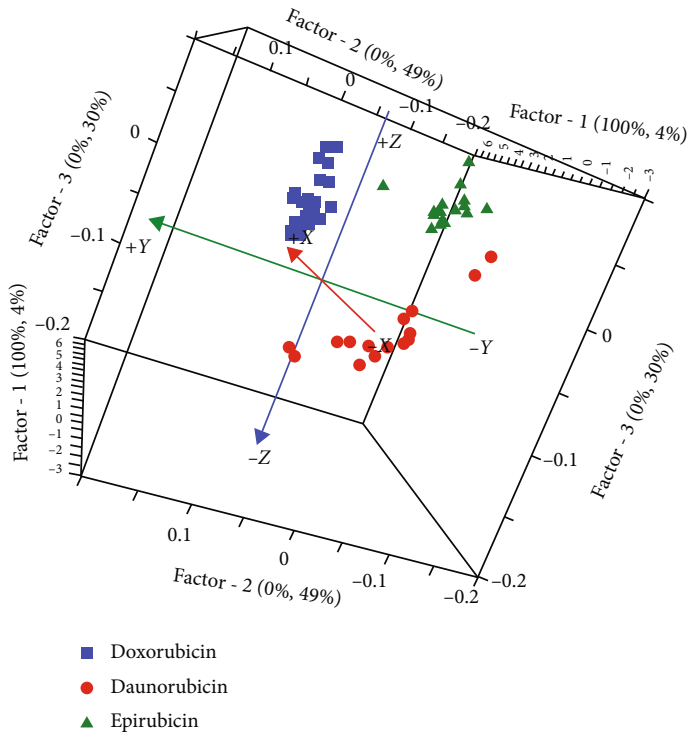

FIgURE 4: 3D-PLS-DA score plot for the UV-visible spectra of the three chemotherapy drugs.

correct classification rate \% CCR, sensitivity, and specificity are the main parameters used to assess the discriminatory power of an analytical method.

The observation of the 3D score plot generated by PLSDA (Figure 4) shows a perfect discrimination between the three groups of chemoterapic drugs. The evaluation of the quality of the constructed model was demonstrated by the high value of $R$-square and the low value of root mean square of calibration RMSEC and crossvalidation RMSECV.

Assessment of the performed models reveals that the correlation coefficient is between $99 \%$ and $98 \%$ for the calibration results and between $98 \%$ and $96 \%$ for the crossvalidation results, whereas the root mean square error of the calibration is between 0.04 and 0.07 and for crossvalidation ranges between 0.06 and 0.08 (Table 1). 
TABLE 1: Statistical parameters of the PLS-DA model.

\begin{tabular}{lcccc}
\hline \multirow{2}{*}{ Label } & \multirow{2}{*}{ Number of latent variable } & \multicolumn{2}{c}{ Calibration } & \multicolumn{2}{c}{ Crossvalidation } \\
& & $R$-square & RMSEC & \multicolumn{2}{c}{$R$-square } & 0.97 \\
Doxorubicin & & 0.99 & 0.05 & 0.08 \\
Daunorubicin & $7 \mathrm{LV}$ & 0.98 & 0.07 & 0.96 \\
Epirubicin & & 0.99 & 0.04 & 0.99 \\
\hline
\end{tabular}

TABLE 2: Confusion matrix for the classification of anthracycline drugs (1: doxorubicin, 2: daunorubicin, and 3: epirubicin) using the PLS-DA method.

\begin{tabular}{|c|c|c|c|c|c|c|c|}
\hline \multirow{2}{*}{ Confusion matrix } & \multicolumn{4}{|c|}{ Actual set } & \multirow[b]{2}{*}{ Specificity \% } & \multirow[b]{2}{*}{ Sensitivity\% } & \multirow[b]{2}{*}{$\% \mathrm{CCR}$} \\
\hline & Label & 1 & 2 & 3 & & & \\
\hline \multirow{3}{*}{ Predicted training set } & 1 & 22 & 0 & 0 & & & \\
\hline & 2 & 0 & 15 & 0 & 100 & 100 & 100 \\
\hline & 3 & 0 & 1 & 15 & & & \\
\hline
\end{tabular}

TABle 3: Performance results of the quantification models developed for all concentration ranges of the three drugs.

\begin{tabular}{lccc}
\hline & Doxorubicin & Daunorubicin & Epirubicin \\
\hline Concentration range $\mathrm{mg} / \mathrm{mL}$ & $0.001-0.064$ & $0.006-0.077$ & $0.006-0.083$ \\
Spectral pretreatment & Raw & Raw & Raw \\
Number of variables latent & 1 & 1 & 1 \\
$R$-square of calibration & 0.99 & 0.99 & 0.99 \\
$R$-square of crossvalidation & 0.99 & 0.99 & 0.99 \\
RMSECV & 0.00037 & 0.00179 & 0.00125 \\
RMSEC & 0.00033 & 0.00162 & 0.00089 \\
\hline
\end{tabular}

Eventually, the results were displayed in the form of confusion matrices, enabling the estimation of the correct classification rate, specificity, and sensitivity of the discrimination achieved across the test set.

According to the confusion matrix (Table 2), it can be seen that all individuals belong to their class with a CCR, specificity, and selectivity of $100 \%$. The validation of this classification model by crossvalidation shows a high capacity in the classification of the three drugs of the anthracycline family with a classification accuracy that reaches $100 \%$. Despite the similarities in structure between doxorubicin, epirubicin, and daunorubicin, since they belong to the same family of anthracyclines and anticancer drugs (56), the combination of UV-visible with PLS-DA was able to differentiate very clearly between these three drug formulations.

The combination of UV-visible spectral data with the PLS-DA method provides powerful results for the discrimination of the three drugs regarding specificity, sensitivity, and CCR. These results are generally consistent with other studies that have used factorial discriminant analysis (PCAFDA) in combination with RAMAN and infrared spectroscopy [27]. Other studies also show a high capacity of the combination of PLS-DA with spectroscopic methods for the discrimination of drugs used in chemotherapy. Another study also shows a high capacity of the combination of PLS-DA with Raman spectroscopy for the discrimination of antineoplastic taxane drugs $[15,21]$.
3.3. Quantitative Analysis. In order to optimize the quantification models of the three drugs of the anthracycline family, a PLS regression method was applied to the UV-visible results. Numerous pretreatments were applied for the construction of the PLS models. The best quantification models were chosen on the basis of statistical parameters, and the best patterns are those having the lowest root mean square error of calibration (RMSEC), the lowest root mean square error of crossvalidation (RMSECV), and the highest $R$ -square.

This table presents the performance parameters of the PLS quantification models and the number of latent variables (LVs) used to build the PLS models. It provides also information on the predictive performance results, including the coefficient of determination $\left(R^{2}\right)$ for calibration and crossvalidation and errors (RMSECV) (RMSEC).

The results found by PLS regression show high performance for the quantification of the three drugs using the first latent variable that represents $100 \%$ of the total variability of the data set. This performance is explained by the low value of RMSECV and RMSEC and the high value of $R$-square (Table 3). Therefore, the high value of $R$-square indicates strong linearity between the reference and predicted values as shown in Figure 5.

These results found are adequate with the results found by Makki et al., which shows an $R$-square of 0.99 and RMSECV ranging between 0.0127 and 0.0220 [27]. 


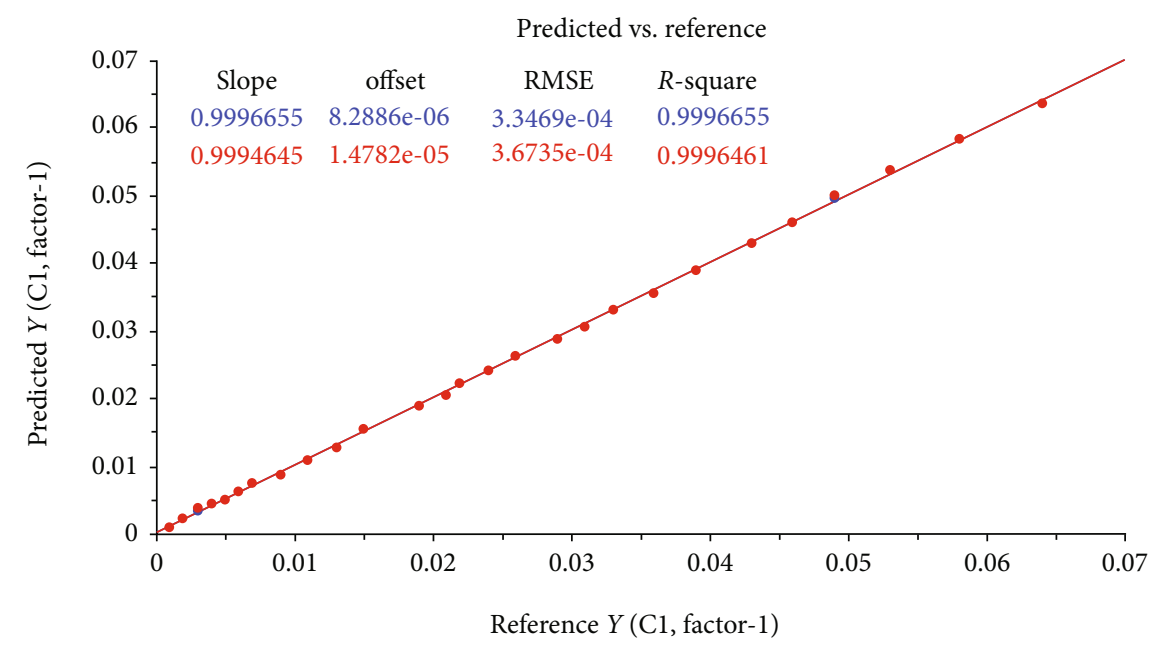

(a)

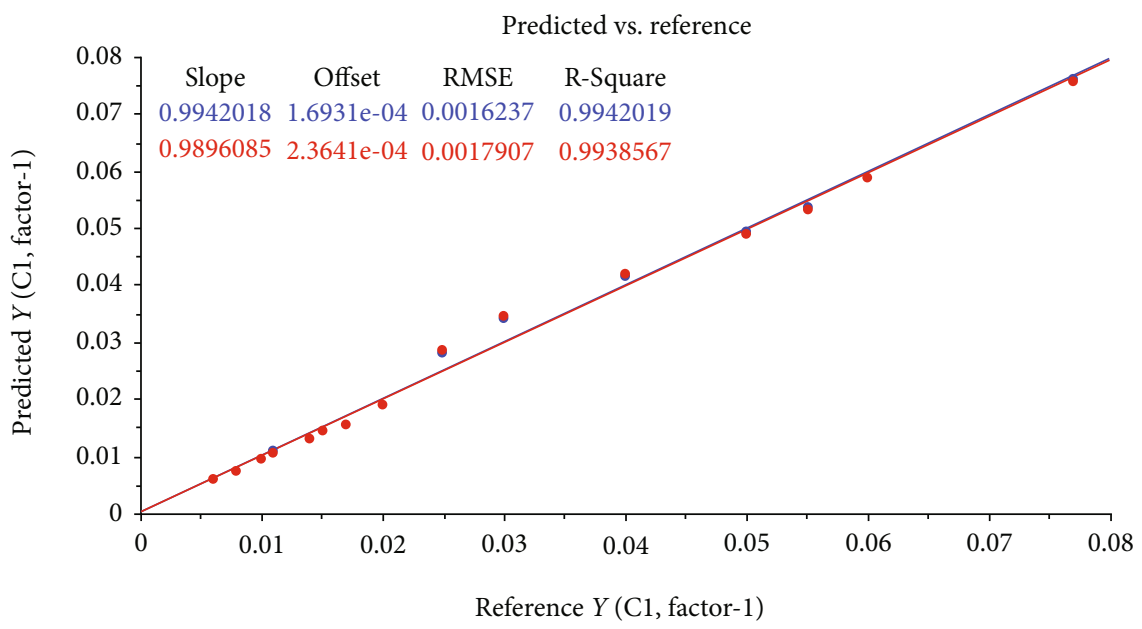

(b)

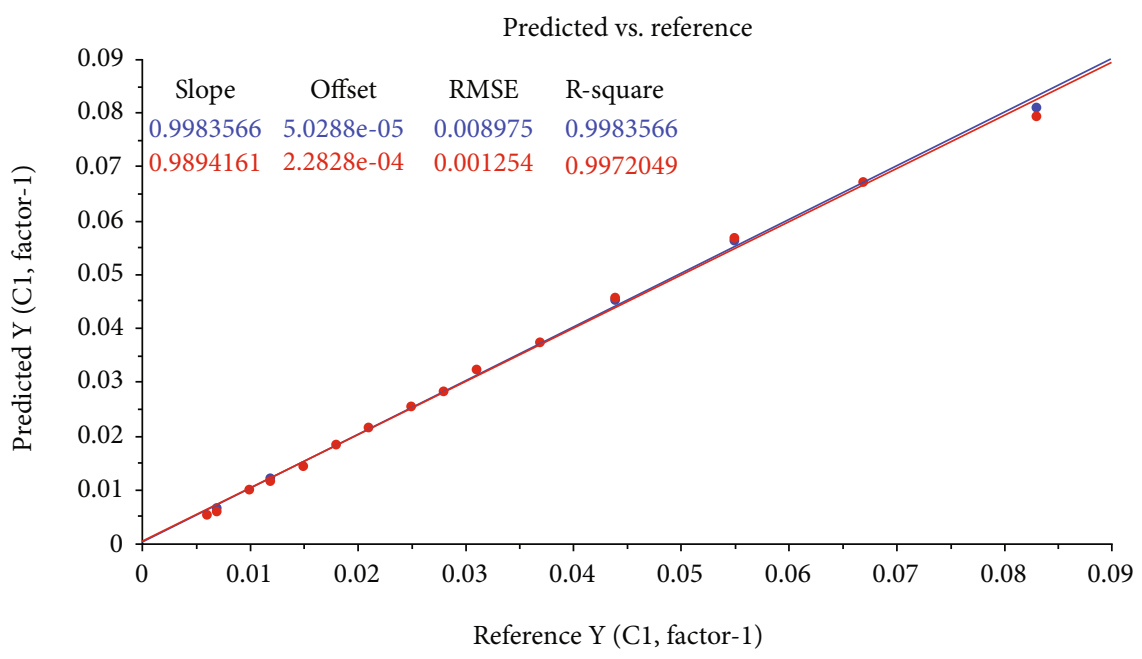

(c)

Figure 5: Actual values vs. predicted values using PLS regression ((a) doxorubicin, (b) daunorubicin, and (c) epirubicin).

This study confirms the qualitative and quantitative capability of the combination of UV-visible spectroscopy and chemometric models to identify and verify cytotoxic drug preparations. Total discrimination was observed over the entire range of therapeutic concentrations, even for low concentrations, and no samples were misclassified. Strong 
linearity was observed for the quantification models over the entire concentration range. In light of these results, the use of UV-visible spectroscopy using chemometric methods is an excellent approach to ensure the nature of the drug prior to administration to patients.

Besides the analytical performance, the robustness of the instrument and its simplicity of use constitute favorable arguments for implementing the UV-visible portable scanner. With regard to the expected results, it will be valuable to expand this trial to additional drugs and containers used to deliver drug therapy in order to enhance safety management in the clinical drug preparation.

\section{Conclusion}

In this study, a rapid qualitative and quantitative approach has been developed for the discrimination and quantification of the content of three drugs used in oncology. The combination of UV-visible spectroscopy and chemometric methods shows high performance for the discrimination between the three drugs with a sensitivity and specificity of $100 \%$.

The application of PLS regression also shows a high capacity in the quantification of these three drugs, and this capacity is explained by the low values of RMSECV and RMSEC as well as the high value of $R$-square.

As a general conclusion, the coupling of UV-visible spectra and suitable chemometric analysis tools, in particular PLS regression and PLS-DA, has demonstrated their efficiency for the discrimination and quantification of doxorubicin, daunorubicin, and epirubicin.

Similar multivariate models are strongly recommended as a clean, rapid, and reliable means of assessing the quality control of clinical chemotherapeutic preparations.

\section{Data Availability}

The data used to establish this research are available on request from the corresponding author.

\section{Conflicts of Interest}

The author (s) declared no potential conflicts of interest with respect to the research, authorship, and/or publication of this article.

\section{Acknowledgments}

The author(s) received no financial support for the research, authorship, and/or publication of this article.

\section{References}

[1] J. Ferlay, H.-R. Shin, F. Bray, D. Forman, C. Mathers, and D. M. Parkin, "Estimates of worldwide burden of cancer in 2008: GLOBOCAN 2008," International Journal of Cancer, vol. 127, no. 12, pp. 2893-2917, 2010.

[2] S. Douedi and M. P. Carson, Anthracycline Medications (Doxorubicin), Stat Pearls Publishing, Treasure Island (FL), Stat Pearls, 2020.
[3] N. Wenningmann, M. Knapp, A. Ande, T. R. Vaidya, and S. Ait-Oudhia, "Insights into doxorubicin-induced cardiotoxicity: molecular mechanisms, preventive strategies, and early monitoring," Molecular Pharmacology, vol. 96, no. 2, pp. 219-232, 2019.

[4] Centralized chemotherapy preparation: April 2012 - Pharmacy Purchasing \& Products Magazinehttps://www.pppmag.com/ article/1087.

[5] "ASHP guidelines on quality assurance for pharmacy-prepared sterile products," http://www.ashpmedia.org/softchalk/softchalk_ leadsurveyorstrainingintro/1\%20PrepGdlQualAssurSterile.pdf.

[6] S. Savelli, M. Roche, C. Curti et al., "Methods to control anticancer chemotherapy preparations ranked by risk analysis," Pharmazie, vol. 5, pp. 251-259, 2018.

[7] S. Nussbaumer, P. Bonnabry, J.-L. Veuthey, and S. FleurySouverain, "Analysis of anticancer drugs: a review," Talanta, vol. 85, no. 5, pp. 2265-2289, 2011.

[8] C. Bazin, B. Cassard, E. Caudron, P. Prognon, and L. Havard, "Comparative analysis of methods for real-time analytical control of chemotherapies preparations," International Journal of Pharmaceutics, vol. 494, no. 1, pp. 329-336, 2015.

[9] A. Delmas, J. B. Gordien, J. M. Bernadou et al., "Quantitative and qualitative control of cytotoxic preparations by HPLCUV in a centralized parenteral preparations unit," Journal of Pharmaceutical and Biomedical Analysis, vol. 49, no. 5, pp. 1213-1220, 2009.

[10] V. Castagne, H. Habert, C. Abbara, E. Rudant, and L. Bonhomme-Faivre, "Cytotoxics compounded sterile preparation control by HPLC during a 16-month assessment in a French university hospital: importance of the mixing bags step," Journal of Oncology Pharmacy Practice, vol. 17, no. 3, pp. 191-196, 2011.

[11] A. S. Rodrigues, A. R. Lopes, A. Leão et al., "Development of an analytical methodology for simultaneous determination of vincristine and doxorubicin in pharmaceutical preparations for oncology by HPLC-UV," Journal of Chromatographic Science, vol. 47, no. 5, pp. 387-391, 2009.

[12] I. Bennani, M. A. Chentoufi, A. Cheikh, M. E. Karbane, and M. Bouatia, "Proposal of a simple and rapid method for the chemotherapy preparations analytical control by spectrophotometry UV-Vis method," Journal of Oncology Pharmacy Practice, vol. 27, no. 1, pp. 99-107, 2021.

[13] F. Nardella, M. Beck, P. Collart-Dutilleul et al., “A UV-Raman spectrometry method for quality control of anticancer preparations: Results after 18 months of implementation in hospital pharmacy," International Journal of Pharmaceutics, vol. 499, no. 1-2, pp. 343-350, 2016.

[14] F. Dziopa, G. Galy, S. Bauler et al., "A quantitative and qualitative method to control chemotherapeutic preparations by Fourier transform infrared-ultraviolet spectrophotometry," Journal of Oncology Pharmacy Practice, vol. 19, no. 2, pp. 121-129, 2013.

[15] L. Lê, M. Berge, A. Tfayli, P. Prognon, and E. Caudron, "Discriminative and quantitative analysis of antineoplastic taxane drugs using a handheld Raman spectrometer," BioMed Research International, vol. 2018, pp. 1-7, 2018.

[16] P. Bourget, A. Amin, A. Moriceau, B. Cassard, F. Vidal, and R. Clement, "The Raman spectroscopy (RS): a new tool for the analytical quality control of injectable in health settings. Comparison of RS technique versus HPLC and UV/Vis-FTIR, applied to anthracyclines as anticancer drug," Pathologie Biologie, vol. 60, no. 6, pp. 369-379, 2012. 
[17] A. El Orche, M. Bouatia, H. Labjar, M. Maaouni, and M. Mbarki, "Coupling Mid Infrared Spectroscopy to mathematical and statistical tools for automatic classification, qualification and quantification of Argan oil adulteration," in 2020 IEEE 6th International Conference on Optimization and Applications (ICOA), pp. 1-4, 2020.

[18] A. El Orche, M. Bouatia, S. Yanisse et al., "Evaluation of the capability of horizontal ATR-FTMIR and UV-visible spectroscopy in the discrimination of virgin olive oils from the Moroccan Region of Beni Mellal-Khenifra," Journal of Spectroscopy, vol. 2020, 9 pages, 2020.

[19] O. Elhamdaoui, A. El Orche, A. Cheikh, B. Mojemmi, R. Nejjari, and M. Bouatia, "Development of fast analytical method for the detection and quantification of honey adulteration using vibrational spectroscopy and chemometrics tools," Journal of Analytical Methods in Chemistry, vol. 2020, pp. 1-9, 2020.

[20] X. Li, K. Xu, H. Li, S. Yao, Y. Li, and B. Liang, "Qualitative analysis of chiral alanine by UV-visible-shortwave near infrared diffuse reflectance spectroscopy combined with chemometrics," RSC Advances, vol. 6, no. 10, pp. 8395-8405, 2016.

[21] L. M. M. Lê, A. Tfayli, J. Zhou, P. Prognon, A. Baillet-Guffroy, and E. Caudron, "Discrimination and quantification of two isomeric antineoplastic drugs by rapid and non-invasive analytical control using a handheld Raman spectrometer," Talanta, vol. 161, pp. 320-324, 2016.

[22] C. P. O'Donnell, Ed., Process Analytical Technology for the Food Industry, Springer, New York, 2014.

[23] L. C. Lee, C.-Y. Liong, and A. A. Jemain, "Partial least squaresdiscriminant analysis (PLS-DA) for classification of highdimensional (HD) data: a review of contemporary practice strategies and knowledge gaps," The Analyst, vol. 143, no. 15, pp. 3526-3539, 2018.

[24] A. El Orche, M. Bouatia, and M. Mbarki, "Rapid analytical method to characterize the freshness of olive oils using fluorescence spectroscopy and chemometric algorithms," Journal of Analytical Methods in Chemistry, vol. 2020, pp. 1-9, 2020.

[25] S. Wold, H. Martens, and H. Wold, "The multivariate calibration problem in chemistry solved by the PLS method," in Matrix Pencils, B. Kågström and A. Ruhe, Eds., pp. 286-293, Springer, Berlin, Heidelberg, 1983.

[26] S. de Jong, "SIMPLS: an alternative approach to partial least squares regression," Chemometrics and Intelligent Laboratory Systems, vol. 18, no. 3, pp. 251-263, 1993.

[27] A. A. Makki, F. Bonnier, R. Respaud et al., "Qualitative and quantitative analysis of therapeutic solutions using Raman and infrared spectroscopy," Spectrochimica Acta. Part A, Molecular and Biomolecular Spectroscopy, vol. 218, pp. 97108, 2019. 